\title{
ARSENIC ANTISITE DEFECTS CORRELATIONS IN LOW TEMPERATURE MBE GaAs*
}

\author{
K.P. KORONA \\ Institute of Experimental Physics, Warsaw University \\ Hoża 69, 00-681 Warszawa, Poland
}

GaAs layers grown by molecular beam epitaxy at low temperatures (LT $\mathrm{GaAs})$ have several interesting properties. For example, concentrations up to $2 \times 10^{20} \mathrm{~cm}^{-3}$ of neutral and up to $5 \times 10^{18} \mathrm{~cm}^{-3}$ of positively charged As $\mathrm{Ga}_{\mathrm{a}}$ defects have been determined in as grown layers. It has been observed that electrical transport in LT GaAs is dominated by hopping conductivity. In the as grown and annealed up to $400^{\circ} \mathrm{C}$ layers, experiments show a high mobility of photo-excited electrons up to $20000 \mathrm{~cm}^{2} /(\mathrm{V} \mathrm{s})$ at about $130 \mathrm{~K}$. Taking into account the very high concentration of ionized defects in LT $\mathrm{GaAs}$, the best possible explanation of the measured value of mobility is an assumption that $\mathrm{As}_{\mathrm{Ga}}^{+}$defects interact with ionized acceptors $\mathrm{A}^{-}$. This leads to creation of $\mathrm{As}_{\mathrm{Ga}}^{+}-\mathrm{A}^{-}$dipoles, which do not scatter electrons as efficiently as single ions. It has been shown that the donor-acceptor correlation could be destroyed by illumination which ionizes $\mathrm{As}_{\mathrm{Ga}}$ defects. Also analysis of hopping conductivity suggests the existence of the donor-acceptor correlations in non-illuminated LT GaAs. Numerical calculation of AsGa-acceptors interaction has been done. It shows that at least below $200 \mathrm{~K}$ dipole formation in LT GaAs is possible and could lead to observed increase in mobility.

PACS numbers: 73.61.Ey, 73.50.Yg

\section{Introduction}

Defects correlations in semiconductors are rather rarely studied probably because of two contradictory conditions which should be fulfilled to obtain observable defects correlation: material should have good crystal structure and it should have high concentration of point defects. In poor quality crystals the concentration of extended defects is too high to observe effects originating from the correlation of point defects. In high quality materials, on the other hand, the concentration of point defects is usually too low to induce ordering. It seems that these two conditions are fulfilled by low temperature gallium arsenide (LT GaAs) layers. One of the most important features of LT GaAs is high nonstoichiometry (1.5 at.\% of excess As) [1] which causes a high concentration of point defects and an expansive lattice strain of approximately $0.1 \%$ [2]. Nevertheless samples grown at $200^{\circ} \mathrm{C}$ or

*This work was supported in part by the State Committee for Scientific Research (Republic of Poland) under grant no. 3 P407 02506. 
higher exhibit high crystalline perfection [2]. The concentration of point defects is remarkable. Neutral arsenic antisite $\mathbf{A s}_{\mathrm{Ga}}$ defects dominate having concentration about $10^{20} \mathrm{~cm}^{-3}$. Since the Fermi level is pinned to $\mathrm{As}_{\mathrm{Ga}}^{0 /+}$ level, the number of ionized $\mathrm{As}_{\mathrm{Ga}}^{+}$centers should be equal to the number of acceptors (mainly $\mathrm{V}_{\mathrm{Ga}}$ ). Its concentration $\left(N_{\mathrm{As}_{\mathrm{Ga}}^{+}}\right)$is $3-5 \times 10^{18} \mathrm{~cm}^{-3}[3,4]$ as measured by electron paramagnetic resonance (EPR). Concentration of $\mathrm{V}_{\mathrm{Ga}}$ defects about $10^{18}-10^{19} \mathrm{~cm}^{-3}$ has been estimated by positron annihilation experiment [5]. This amount of defects is sufficiently high to create a deep defect band. It has been shown that in as grown LT GaAs layers hopping conductivity between arsenic antisite defects exists $[3,6]$.

\section{Experimental}

\subsection{Samples}

LT MBE GaAs layers have been grown at temperature $190^{\circ} \mathrm{C}$ under As rich conditions in MIT Lincoln Laboratory by Drs. F.W. Smith and R. Calawa. Layers of 2.0-2.5 $\mu \mathrm{m}$ thickness have been grown on semi-insulating substrate. The samples were annealed in the MBE system at temperatures from 300 to $600^{\circ} \mathrm{C}$. As calculated from the near-infrared optical absorption spectra using Martin's calibration curve [7], the concentration of arsenic antisite defect in as grown samples was $1 \times 10^{2} \mathrm{~cm}^{-3}$, while in samples annealed at $400^{\circ} \mathrm{C}$ its concentration was about five times lower. Annealing at $600^{\circ} \mathrm{C}$ caused a decrease in As $\mathrm{As}_{\mathrm{G}}$ defect concentration to such a level that optical detection was very difficult. Using results of hopping conductivity measurements $N_{\mathrm{As}_{\mathrm{G}_{4}}}$ was estimated to be $9 \times 10^{18} \mathrm{~cm}^{-3}$.

\subsection{Hopping conductivity}

Concentration of arsenic antisite defects $\left(N_{\mathrm{As}_{\mathrm{G}_{4}}} \approx 10^{20} \mathrm{~cm}^{-3}\right)$ in LT GaAs is high enough to create a deep defect band. It has been shown that in as grown LT GaAs layers the hopping conductivity between arsenic antisite defects exists $[3,6]$ (see Fig. 1). In as grown samples hopping dominates even in room temperature. After annealing of the layer, hopping decreases but still could be observed in temperature below $250 \mathrm{~K}$. In higher temperature electrons are thermally excited from $\mathrm{As}_{\mathrm{Ga}}$ to the conduction band and the free electron transport dominates.

According to theory of hopping conductivity, in samples with high impurities concentration it is possible that electrons can move from one center to another by tunneling between them. This conduction mechanism is important when temperature is too low for thermal excitation of electrons to the conduction band. Just below this temperature the following relation is expected [8]:

$$
\sigma=\sigma_{03} \exp \left(\frac{\varepsilon_{3}}{k T}\right),
$$

where $\varepsilon_{3}$ is an average difference of potential energies between initial and final state of tunneling electron. This difference is usually caused by potential fluctuations in crystal. Theoretical line plotted in Fig. 1 shows that relation (1) fits to the experimental data down to $100 \mathrm{~K}$. In lower temperature the conductivity changes more slowly which is in agreement with the theory developed by Mott and Shklovskii $[8,9]$. 


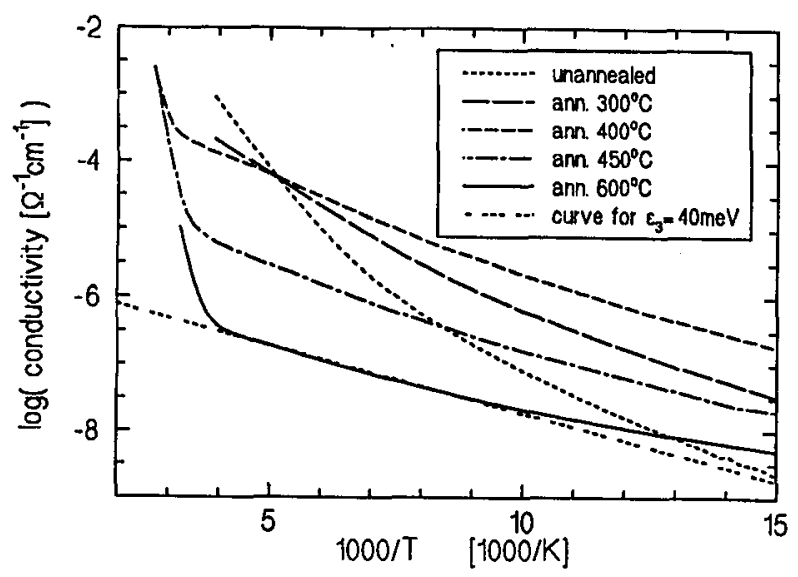

Fig. 1. Conductivity $\sigma$ versus 1000 /temperature measured for samples of LT GaAs annealed at different temperatures. Theoretical line fitted to the lowest experimental curve represents temperature dependence of hopping conductivity.

TABLE

Hopping parameters of LT GaAs layers.

\begin{tabular}{c|c|c|c|l}
\hline \hline$T_{\text {ann }}\left[{ }^{\circ} \mathrm{C}\right]$ & $N_{\mathrm{As}_{\mathrm{G}_{2}}}\left[\mathrm{~cm}^{-3}\right]$ & $\sigma_{03}\left[\mathrm{~cm}^{-1}\right]$ & $\varepsilon_{3}[\mathrm{eV}]$ & \\
\hline unannealed & $1.1 \times 10^{20}$ & 3,7 & 157,195 & 2 samples \\
300 & $7 \times 10^{19}$ & 0.02 & 96 & \\
350 & $5 \times 10^{19}$ & 0.002 & 64 & \\
400 & $2 \times 10^{19}$ & 0.002 & 62 & \\
450 & $1.2 \times 10^{19}$ & $7 \times 10^{-5}$ & 55 & \\
600 & $9 \times 10^{18}$ & $2 \times 10^{-6}$ & 42,44 & 2 samples
\end{tabular}

By fitting Eq. (1) to the experimental data, energies $\varepsilon_{3}$ for all samples were determined (see Table). The energy $\varepsilon_{3}$ can reach $0.2 \mathrm{eV}$ (in as grown material).

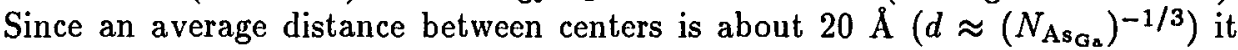
would suggest existence of an electric field $E=10^{6} \mathrm{~V} / \mathrm{cm}$. Another possible explanation is that $\mathrm{As}_{\mathrm{Ga}}^{+}$center interacts with ionized acceptor $\mathrm{A}^{-}$. In order to explain the high value of $\varepsilon_{3}$ the two centers have to lie very closely. It is possible in situation when $\mathrm{As}_{\mathrm{Ga}}^{+}-\mathrm{A}^{-}$correlation exists.

\subsection{Transport in the conduction band}

In the case of hopping conductivity the Hall mobility is infinitesimal, which has been confirmed by the measurements of LT GaAs. However, in higher temperature where transport in conduction band dominates an effective mobility of the order of $10^{3} \mathrm{~cm}^{2} /(\mathrm{V} \mathrm{s})$ was observed (continuous line in Fig. 2). It was due to transport by electrons thermally excited to the conduction band.

In the case of two transport mechanisms effective mobility and concentration 


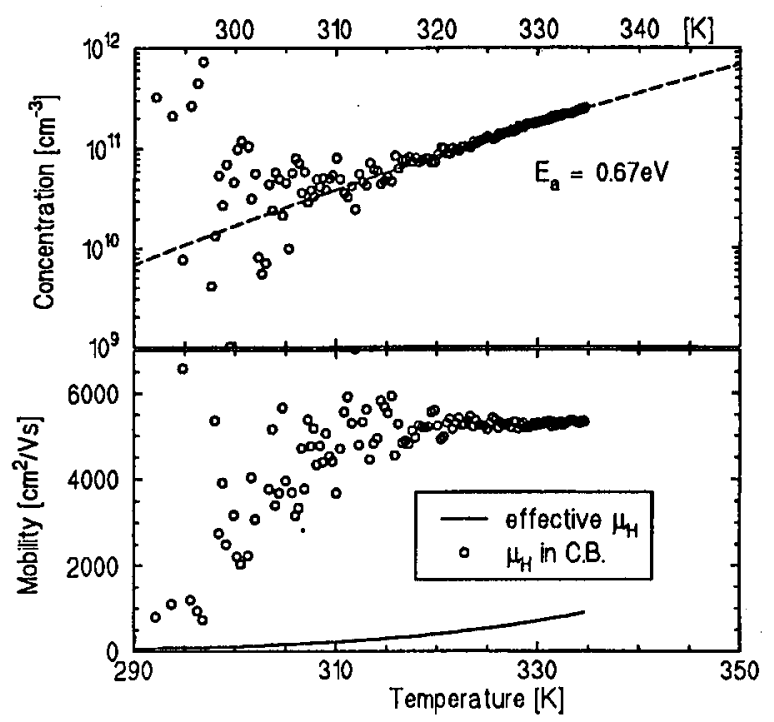

Fig. 2. Hall mobility and concentration of electrons measured for LT GaAs. Dotted line in the upper picture describes thermal activation of electrons from AsGa level. Experimentally measured effective mobility is plotted by continuous line.

are as follows (since $\mu \leq 1 \mathrm{~m}^{2} /(\mathrm{V} \mathrm{s})$ and $B=0.05 \mathrm{~T}$, components containing $\mu^{2} B^{2}$ were neglected):

$$
\mu_{\mathrm{eff}}=\mu \frac{n^{*} \mu \rho_{3} e}{1+n^{*} \mu \rho_{3} e}, \quad n_{\mathrm{eff}}=n \frac{n^{*} \mu^{2} \rho_{3} e}{\left(1+n^{*} \mu \rho_{3} e\right)^{2}},
$$

where $n^{*}, \mu$ - concentration and mobility of excited electrons, $\rho_{3}$ - hopping resistivity. Value of $\rho_{3}$ can be approximated from dependence (1) fitted to the low temperature data. Basing on Eqs. (2) and (3), the Ifall mobility $\mu$ and concentration $n^{*}$ as functions of temperature were calculated (see Fig. 2). Unfortunately, below $310 \mathrm{~K}$ the electron concentration decreased so strongly that it was impossible to calculate mobility. But above this temperature, it seems that the method gives reliable results. For example, concentration of excited electrons is in agreement with assumption that they came from $\mathrm{As}_{\mathrm{Ga}}$ deep level (activation energy $E_{\mathrm{a}}=0.67 \mathrm{eV}$ is lower than expected value $0.75 \mathrm{eV}$ probably due to potential fluctuations in sample). However, the obtained value of mobility $\mu \approx 5000 \mathrm{~cm}^{2} /(\mathrm{V} \mathrm{s})$ is much higher than expected. In the sample with ionized impurity concentration of the order of $10^{19} \mathrm{~cm}^{-3}$ the mobility should be lower than $10^{3} \mathrm{~cm}^{2} /(\mathrm{V} \mathrm{s})$. One of the possible explanation is that positively and negatively charged impurities are correlated which leads to decrease in electron scattering.

\subsection{Electrical transport under illumination}

Photoconductivity measurement of LT GaAs shows (see Fig. 3) that PC spectrum was dominated by photo-ionization band at $h \nu=0.8-1.5 \mathrm{eV}$ which was identified as photoionization spectrum of EL2 defect $\left(\mathrm{As}_{\mathrm{Ga}}\right)$ [10]. In low tempera- 
ture $(12 \mathrm{~K})$, this band could be quenched by illumination with white or monochromatic $h \nu=1.3 \mathrm{eV}$ light. The band recovers in temperature higher than $130 \mathrm{~K}$. It means that metastable behavior of this band is the same as behavior of EL2 $\left(\mathrm{As}_{\mathrm{Ga}}\right)$ photoionization band in standard semi-insulating (SI) GaAs.

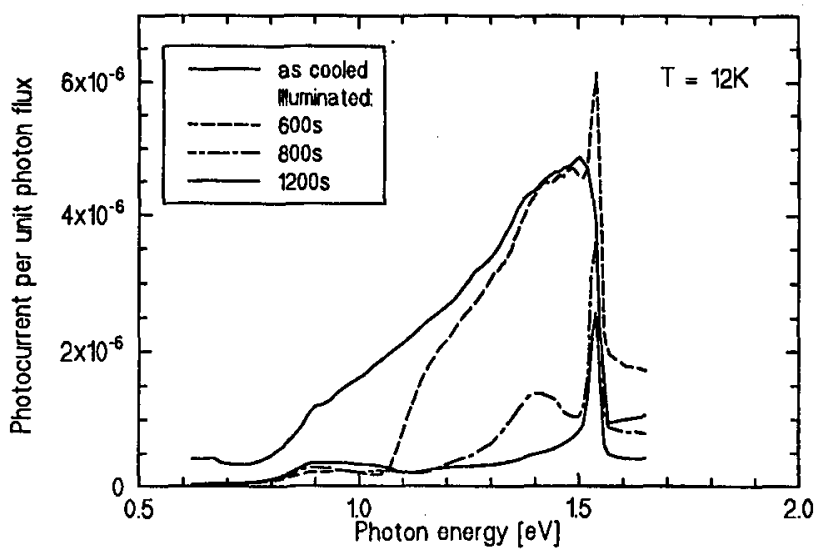

Fig. 3. Photocurrent spectra of LT GaAs measured at $T=12 \mathrm{~K}$ before and after illumination with light of $h \nu=1.3 \mathrm{eV}$.

As was shown in Fig. 2, below room temperature it was impossible to measure the Hall effect on LT GaAs. However, after illumination of layers, a dramatic increase in mobility was observed. It was due to the transport by electrons excited to the conduction band. In the experiment, monochromatic light with energy $h \nu=$ $1.9 \mathrm{eV}$ and $h \nu=1.3 \mathrm{eV}$ was used. Light of $h \nu=1.9 \mathrm{eV}$ creates electron-hole pairs whereas $1.3 \mathrm{eV}$ light excites electrons from $\mathrm{As}_{\mathrm{Ga}}$ defects to the conduction band.

Based on measured dependence of $\mu_{\mathrm{eff}}$ and $n_{\mathrm{eff}}$ versus temperature, using Eqs. (2) and (3), the excited carriers concentration $n^{*}$ and their mobility $\mu$ as functions of temperature were calculated. It was taken into account that $\rho_{3}$ value was the same as in the dark. Figure $4 \mathrm{~A}$ shows dependence of the Hall concentration of photo-excited electrons versus temperature. The activation energy of the photo-excited carriers concentration was determined as $E_{\mathrm{a}}=0.07 \pm 0.02 \mathrm{eV}$. This value corresponds to the activation energy of electron capture cross-section of EL2 $\left(\mathrm{As}_{\mathrm{Ga}}\right) E_{\mathrm{b}}=0.066 \mathrm{eV}$ [11], which suggests that electron lifetime $\tau_{n}$ is governed by the capture by $\mathrm{As}_{\mathrm{Ga}}$ defects.

The mobility of the excited carriers versus temperature for as grown sample was shown in Fig. 4B. Using $1.9 \mathrm{eV}$ light, the mobility of the photo-excited electron having maximum at temperature $130 \mathrm{~K}$ equal to $\mu=20000 \mathrm{~cm}^{2} /(\mathrm{V} \mathrm{s})$ was observed. Absorption coefficient for the $h \nu=1.9 \mathrm{eV}$ light is $\alpha=3.7 \mu \mathrm{m}^{-1}$, electron diffusion length in LT GaAs is about $L_{\mathrm{D}}=0.1 \mu \mathrm{m}$, therefore nearly all photo-excited electrons should stay in the LT GaAs layer. While illuminating with the $1.3 \mathrm{eV}$ light, analogous results for mobility were obtained (see Fig. 4B), although for this light the sample is more transparent and part of it (about 2\%) 


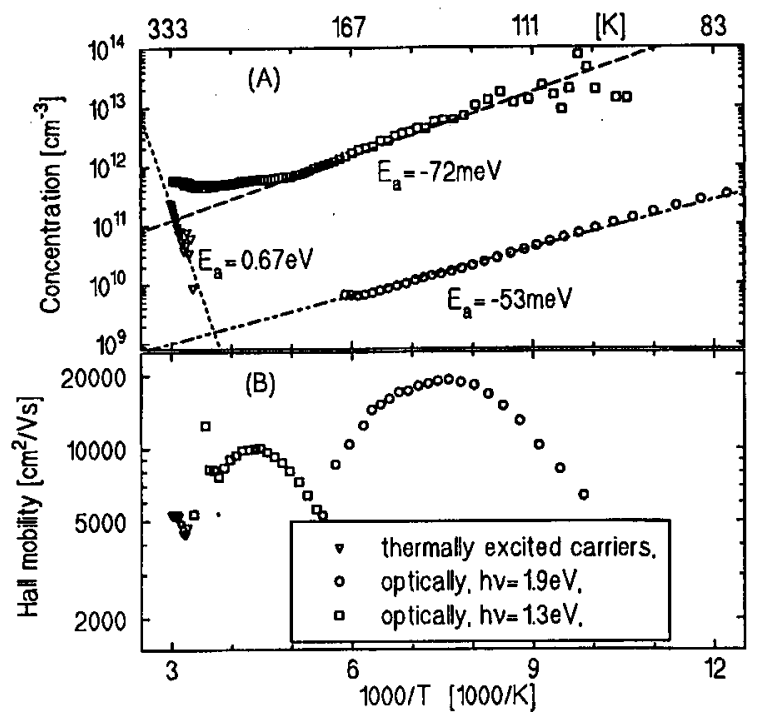

Fig. 4. Results of measurements of Hall effect: (A) Hall concentration and (B) Hall mobility of electrons excited to the conduction band.

is absorbed in the substrate which complicates the effect. It can be noticed that below certain temperature the mobility quickly decreases. It is probably connected with a strange effect of lowering of mobility by intensive illumination which occurs in low temperatures. The effect is stronger for the $1.3 \mathrm{eV}$ light than for the $1.9 \mathrm{eV}$ light. Therefore, for the further analysis peak value of the mobility should be taken rather than the full curve. It is worth noticing that the mobility value $\mu=20000 \mathrm{~cm}^{2} /(\mathrm{V} \mathrm{s})$ is nearly two orders of magnitude higher than expected for material with $10^{20} \mathrm{~cm}^{-3}$ defects and about $10^{19} \mathrm{~cm}^{-3}$ ionized centers.

It is proposed that both these effects, high mobility value and the lowering of mobility by intensive illumination, could be connected with spatial correlation of ionized centers. Under weak illumination the correlated ionized impurities very weakly scatter electrons. Under intensive illumination the correlations are destroyed and scattering increases. This observation gives opportunity to gather more information about the postulated correlations. For this purpose an experiment with two beams of light was performed.

\subsection{Two beam experiment}

As it has been described above, in temperature range 110-160 K under illumination of $1.9 \mathrm{eV}$ light high mobility of electrons in LT GaAs has been observed. It was suggested that electron scattering in the sample is weak because of correlation between charged centers leading to formation of $\mathrm{As}_{\mathrm{Ga}}^{+}-\mathrm{A}^{-}$pairs (see Fig. 6). Since number of acceptors is much lower than number of $A s_{\mathrm{Ga}}$ defects and electrons can migrate via hopping between $\mathrm{As}_{\mathrm{Ga}}$ defects, it is possible that the $\mathrm{As}_{\mathrm{Ga}}$ defect closest to the acceptor would be chosen and positively ionized. When the material with correlated donor-acceptor pairs is illuminated by light which can ionize de- 


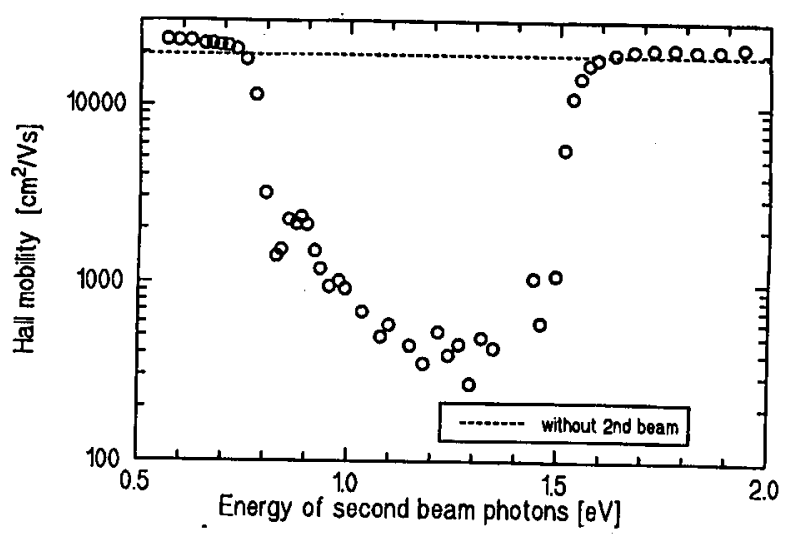

Fig. 5. Hall mobility measured under illumination with two beams of light at $T=$ $120 \mathrm{~K}$. The first beam, $h \nu=1.9 \mathrm{eV}$, generates electrons, the second can also ionize deep centers.

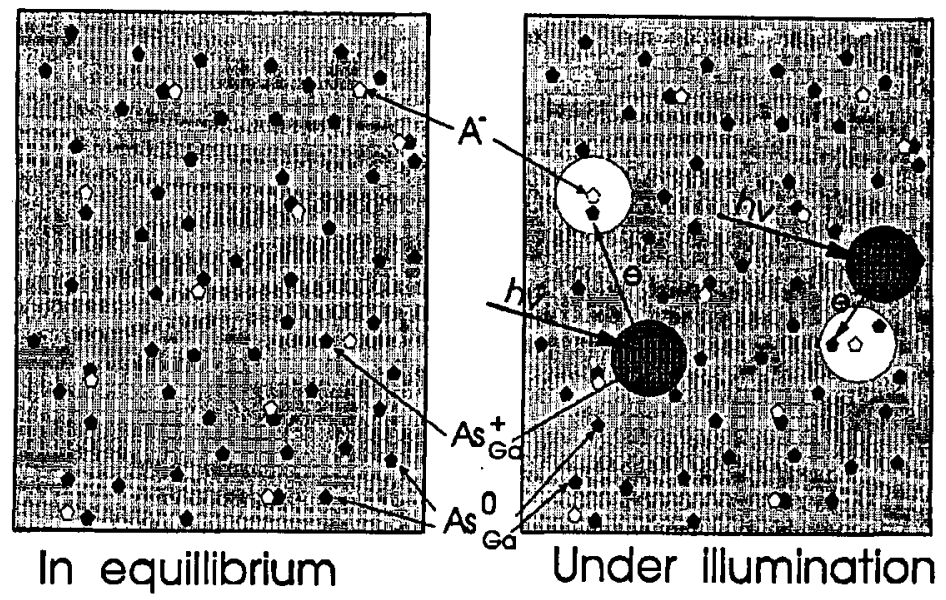

Fig. 6. Model of defect correlation: left, in equilibrium ionized impurities $\mathrm{As}_{\mathrm{Ga}}^{+}$and acceptors $\mathrm{A}^{-}$form dipoles; right, under illumination, light destroys dipoles.

fects (see Fig. 6 right), it is possible that part of uncorrelated As $_{G_{a}}$ defects will be ionized and that excited electrons will recombine on previously correlated centers producing unpaired acceptors. Therefore, instead of the dipoles a great number of unscreened ionized impurities is created which effectively scatters carriers.

Results of this experiment, plotted in Fig. 5 shows that indeed illumination with second beam changes electron mobility. Carriers were excited using low intensity $1.9 \mathrm{eV}$ light. Second beam was swept from 0.5 to $2.0 \mathrm{eV}$. A wide band ranging from 0.8 to $1.5 \mathrm{eV}$ has been observed. The change of mobility has been remarkable, nearly two orders of magnitude. Comparison with Fig. 3 shows that this "low mobility band" corresponds to photoionization band of $\mathrm{As}_{\mathrm{Ga}}$ defect, thus the effect 
is obviously connected with $\mathrm{As}_{\mathrm{Ga}}$ ionization. The Hall concentration of electrons under this illumination is about $10^{11} \mathrm{~cm}^{-3}$. The change of ionized defects concentration should be equal to this number. Therefore it is impossible that increase in scattering was caused by increase in ionized defects concentration. Simultaneously, changes in ordering could be very significant, because time of repairing of correlation is much longer than the electron lifetime. Concluding, this experiment directly confirms existence of ionized impurity correlations in LT GaAs and shows crucial role of $\mathrm{As}_{\mathrm{Ga}_{\mathrm{a}}}$ in these correlations.

\section{Numerical calculations}

From experiment, it is known that in LT GaAs correlations of ionized defects exist which causes an increase in electron mobility. It was also proved that $\mathrm{As}_{\mathrm{Ga}}$ defect plays crucial role in this ordering. J. Mycielski [12] has shown that in $\mathrm{HgFeSe}$ an ordering in space of ionized donors occurs which has a dramatic effect on scattering of free carriers. In this model charged centers should form a superlattice of equally spaced centers. Unfortunately, liquifying temperature of this system is rather low, $T_{0} \approx 40 \mathrm{~K}$. The idea of correlation which is described in this paper is different. It assumes that negatively charged acceptors and positively charged $\mathrm{As}_{\mathrm{Ga}}^{+}$defects form pairs.

Let us take into account that there is an acceptor $\mathrm{A}^{-}$surrounded by $\mathrm{As}_{\mathrm{Ga}}$ defects. One of these defects should be ionized. Taking into account the Coulomb interaction between charged centers, probability of existence of ionized $\mathrm{As}_{\mathrm{Ga}}^{+}$defect at a distance $r_{i}$ from the acceptor is given by equation

$$
P_{\mathrm{As}_{\mathrm{G}_{4}}^{+}}\left(r_{i}\right)=A P_{\mathrm{As}_{\mathrm{G}_{\mathrm{a}}}}\left(r_{i}\right) \exp \left(-\frac{e^{2}}{4 \pi \varepsilon_{0} \varepsilon_{\mathrm{r}} r_{i} k_{\mathrm{B}} T}\right),
$$

where $P_{\mathrm{As}_{\mathrm{Ga}}}\left(r_{i}\right)$ is the probability of existence of $A s_{\mathrm{Ga}_{a}}$ defect at a distance $r_{i}$ from the acceptor and $A$ is a normalization factor defined by the equation

$$
\sum_{r_{i} \in V} P_{\mathrm{As}_{\mathrm{G}_{i}}^{+}}\left(r_{i}\right)=1
$$

where $V$ is the volume of crystal containing one acceptor.

If we take into account random distribution of $\mathrm{As}_{\mathrm{Ga}}$ defect the probability $P_{\mathrm{As}_{\mathrm{Ga}}}\left(r_{i}\right)$ is

$$
P_{\mathrm{As}_{\mathrm{G}_{\mathrm{a}}}}\left(r_{i}\right)=d^{3} n_{\mathrm{s}}\left(r_{i}\right) N_{\mathrm{As}_{\mathrm{G}_{\mathrm{a}}}} / 4 \text {, }
$$

where $d$ is lattice constant and $n_{\mathrm{s}}\left(r_{i}\right)$ is number of gallium sites at the distance $r_{i}$ from the acceptor. Distribution (6) could be changed for example by As diffusion during growth.

Based on Eqs. (4), (5), (6), probability distributions $P_{\mathrm{As}_{\mathrm{G}_{a}^{+}}^{+}}(r)$ for different temperatures were calculated. Analysis of the results (see Fig. 7 ) shows the significant increase in probability $P_{\mathrm{As}_{\mathrm{Ga}}^{+}}(r)$ for small radius $r$ in low temperature. We can conclude that in temperature below $200-300 \mathrm{~K}$ the probability of placing ionized $\mathrm{As}_{\mathrm{Ga}}^{+}$defect closely to the acceptor is very high so the postulated correlations should exist. In temperature $400 \mathrm{~K}$ or higher correlations are dissolved. Curve plotted for $T=\infty$ is proportional to $P_{\mathrm{As}_{\mathrm{Ga}}}(r)$ distribution. 


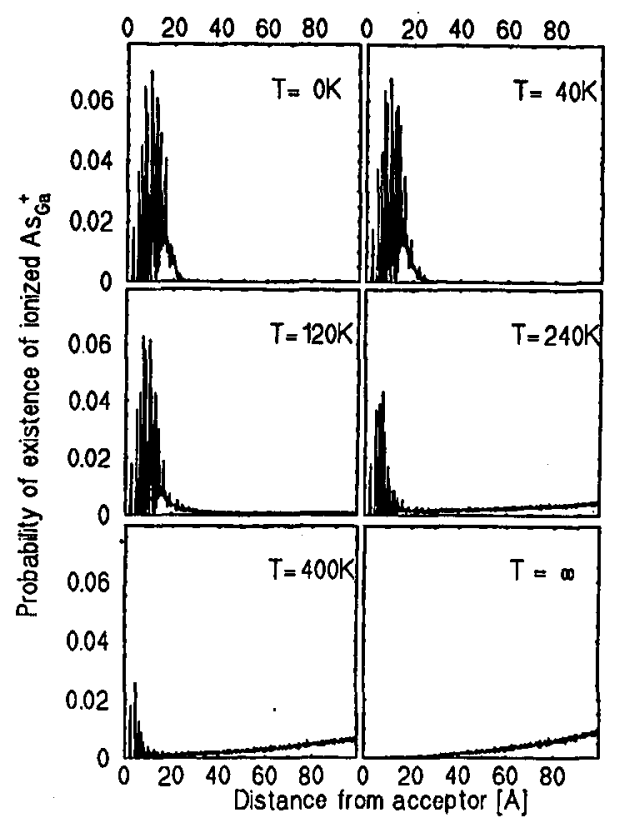

Fig. 7. Probability of the ionized $\mathrm{As}_{\mathrm{Ga}}^{+}$defect existence vs. distance from attracting it acceptor, calculated for six temperatures.

It is important to compare the experimentally measured mobility with the theoretical calculations based on the correlation model (see Fig. 8). The following scattering mechanisms were taken into account for calculation of electron mobility in LT GaAs: optical and acoustic phonon scattering, scattering on ionized impurities and scattering on dipoles. Since free electron concentration is too low for effective screening of charged centers, the Conwell-Weisskopf formula [13] has been applied for the scattering on ionized impurities. The effect of the dipole scattering was calculated using Dimitrov formula [14]. Based on the Erginsoy formula [15] it has been estimated that scattering on deep neutral impurities could be neglected. Calculations have been done for two acceptor concentrations $N_{\mathrm{A}}=1 \times 10^{18} \mathrm{~cm}^{-3}$ and $N_{\mathrm{A}}=5 \times 10^{18} \mathrm{~cm}^{-3}$. Three models have been checked. The first one assumes that charged centers in the sample are not correlated and $N_{\mathrm{As}_{\mathrm{G} a}^{+}}=N_{\mathrm{A}}$. The second model stands that ionized $\mathrm{As}_{\mathrm{Ga}}^{+}$defects are distributed according to Eqs. (4), (5), (6). The third takes into account very weak As diffusion during growth, therefore the distribution (6) is replaced by the distribution calculated using numerical simulation of As diffusion. This diffusion allows arsenic ions to be moved over few angstroms distances. It means that in the crystal there are built-in correlations between arsenic antisites and acceptors (although they are very weak).

The results of numerical calculations are compared with experimental data in Fig. 8. As is seen, in the case of scattering on uncorrelated impurities the calculation results strongly differ from experimental data. The discrepancy is higher than order of magnitude. The models with correlations fit much better. The model 


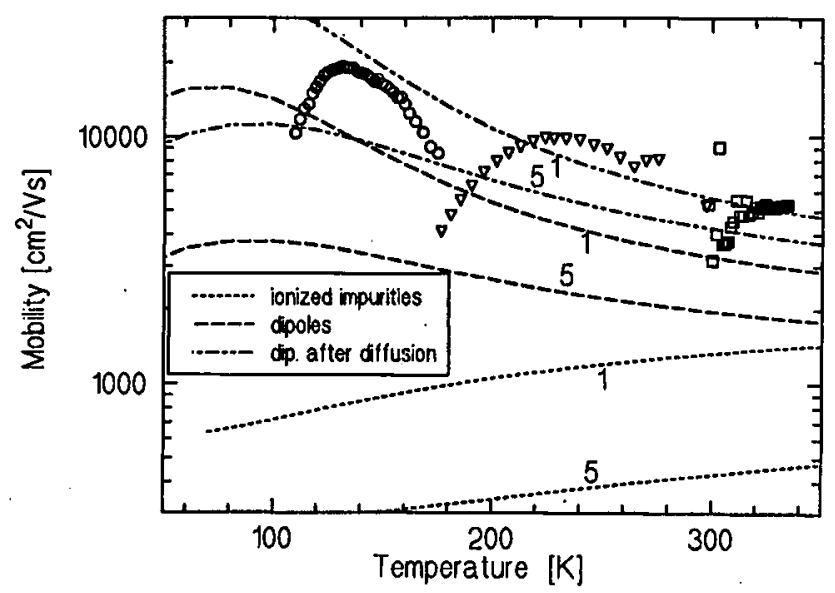

Fig. 8. Comparison of experimental data (plotted as circles, squares and triangles) and calculation results (dotted lines). Lines calculated for acceptor concentration $N_{\mathrm{A}}=$ $1 \times 10^{18} \mathrm{~cm}^{-3}$ are labeled " 1 ", for $N_{\mathrm{A}}=5 \times 10^{18} \mathrm{~cm}^{-3}$ are labeled " 5 ".

taking into account As diffusion during growth is the best, but the model with random $\mathrm{As}_{\mathrm{Ga}}$ distribution is also quite good. Thus, it cannot be distinguished whether the correlations are partially built-in or they are formed only after growth. These built-in As correlations could be treated as a starting point for As precipitates which have been observed in annealed samples [16].

\section{Conclusions}

In spite of a great concentration of defects in LT GaAs, carriers excited to the conduction band have high mobility (nearly $20000 \mathrm{~cm}^{2} /(\mathrm{V} \mathrm{s})$ at $130 \mathrm{~K}$ ). Such high mobility suggests that ionized impurities are not randomly distributed, but their positions are correlated. It was assumed that mainly the $\mathrm{As}_{\mathrm{Ga}}$ defects nearest to acceptors are ionized. Numerical calculations proved that formation of the postulated correlation is possible. The $\mathrm{As}_{\mathrm{Ga}}^{+}-\mathrm{A}^{-}$pairs should be spontaneously created below room temperature due to the Coulomb interaction. It has been shown that this effect could explain the experimentally observed phenomena.

\section{Acknowledgments}

The author would like to thank E.R. Weber from University of California for providing LT GaAs samples. Fruitful discussions with M. Kamińska and J.M. Baranowski (both from Warsaw University) are greatly acknowledged.

\section{References}

[1] F. Smith, A. Calawa, C.-L. Chen, M. Manfra, L. Mahoney, Electron. Device. Lett. 9, 77 (1988).

[2] A. Claverie, Z. Liliental-Weber, Mater. Sci. Eng. B 22, 45 (1993). 
[3] M. Kamińska, E.R. Weber, Mater. Sci. Forum 83-87, 1033 (1992).

[4] H.J. von Bardeleben, M.O. Manasreh, D.C. Look, K.R. Evans, C.E. Stutz, Phys. Rev. B 45, 3372 (1992).

[5] P. Hautojärvi, J. Mäkinen, S. Palko, K. Saarinen, C. Corbel, L. Liszkay, Mater. Sci. Eng. B 22, 16 (1993).

[6] J. Muszalski, A. Babiński, K.P. Korona, E. Kaminiska, A. Piotrowska, M. Kaminiska, E.R. Weber, Acta Phys. Pol. A 80, 413 (1991).

[7] G.M. Martin, Appl. Phys. Lett. 39, 747 (1981).

[8] B.I. Shklovskii, A.L. Efros, Properties of Doped Semiconductors, Springer-Verlag, Berlin 1984.

[9] N.F. Mott, Philos. Mag. 19, 835 (1969).

[10] M. Kaminiska, M. Skowronski, J. Lagowski, J.M. Parsey, H.C. Gatos, Appl. Phys. Lett. 43, 302 (1983).

[11] A. Mittoneau, A. Mircea, G.M. Martin, D. Pons, Rev. Phys. Appl. 14, 853 (1979).

[12] J. Mycielski, Solid State Commun. 60, 165 (1986).

[13] E. Conwell, V.F. Weisskopf, Phys. Rev. 77, 388 (1950).

[14] H.D. Dimitrov, J. Phys. Chem. Solids 37, 825 (1976).

[15] T.C. McGill, R. Baron, Phys. Rev. B 11, 5208 (1975).

[16] A.C. Warren, J.M. Woodal, J.L. Freeouf, D. Grischkosky, D.T. McInturff, M.R. Melloch, N. Otsuka, Appl. Phys. Lett. 57, 1331 (1990). 